\title{
Approximations for the likelihood ratio for continuous multi-parameter stochastic processes
}

\author{
Rob Luesink*and Arunabha Bagchi ${ }^{\dagger}$ \\ 32nd CDC \\ December 15-17, 1993 \\ San Antonio, Texas
}

\begin{abstract}
Based on finitely additive white noise theory, we may derive the likelihood ratio for random variables with values in any Hilbert space [5], [10]. This includes stochastic processes, defined on an one- or multi-dimensional continuous-parameter bounded domain. In certain circumstances, the likelihood ratio for continuous processes may be computed directly. In general however, we will have to approximate the likelihood ratio. In this paper approximations for the likelihood ratios for continuous-parameter processes will be studied. Starting from a sequence of finite dimensional projection operators in the Hilbert space, strongly converging to identity, we show that the likelihood ratios for the projected processes converge to the likelihood ratio for the original process. Discretisation of the atochastic process turns out to be one of the possibilities for such approximations. The discretization method is expected to give good results for signals satisfying elliptic PDE's, because discretisation of these processes leads to Nearest Neighbor models [1], [2], [9], for which the likelihood ratio has been obtained in [12].
\end{abstract}

\section{Introduction}

In finitely additive white noise theory, one- and multi-parameter white noise processes are considered to be 'weak random variables' mapping the original probability space $(\Omega, A, P)$ into a Hilbert space. For continuous-parameter processes, this Hilbert space should be a function space, usually taken to be $L_{2}(D)$, the space of functions on the parameter domain $D$ with finite energy. Thus defined, white noise processes are in general not 'ran-

-Rob Lueaink, Mathematica Department, University of Limburg, P.O.Box 616, 6200 MD Masetricht, The Netherlands, rob.lueaink Omath Qrulimburg.nl.

tArunabha Barchi, Department of Applied Mathematics, University of Twente, P.O.Box 217, 7500 AE Enschede, The Netherlands, berchiOmath.utwente.nl. dom variables' in classical sense since the measure induced by the process in the image space is not ensured to be countably additive over the Borel $\sigma$-algebra in the image space.

The main advantage of finitely additive white noise theory over the classical theory of stochastic processes, is that it enables us to define white noise in such a way that it can really be seen as the natural extension of the notion of discreteparameter white noise. The results obtained in this theory are precise limits of corresponding results for band-limited processes and no correction terms (like Wong-Zakai correction) have to be introduced.

One drawback of white noise theory is that we have to work with measures that are only finitely additive. This lack of countable additivity however can be compensated by using the topological structure of the Hilbert space. For more about finitely additive white noise, we refer to $[6],[5]$, $[7],[8],[3]$ and $[4]$.

Based on finitely additive measures, it is quite easy to determine the likelihood ratio for stochastic processes, as is pointed out in section 3. One requirement for the process is that it is 'signal plus noise', where the signal is a physical random variable. The derivation of the likelihood ratio is independent of the fact whether the process has a discrete or continuous parameter, or whether the parameter is one- (mostly time) or multi-dimensional [6], [10]. This expression for the likelihood ratio however contains terms, which in general cannot be directly expressed in computable forms. Situations for which this problem is solvable are the case where the process is cyclic, and the case where we are able to derive the 'one-directional filter' (filtering in one direction, smoothing in the other directions of the parameter domain of the process) [10]. An example for which this may solve the problem is a generalisation of Wong's two-parameter model [11].

In general however, we are not able to compute the likelihood ratio for the continuous-parameter 
problem. Therefore, we may look for 'good' approximations. In this paper we determine a class of approximations which are unbiased in a way we define later in this paper. Moreover, discretization of the continuous-parameter process turns out to be one of the possibilities to approximate the likelihood ratio. Especially for distributed parameter problems defined by means of elliptic PDE, discretization gives a very simple way to approximate the likelihood ratio. Discretization of elliptic PDE results in a Nearest Neighbor model (NNM) $[1],[2],[9]$, for which the likelihood ratio has been obtained in [12].

\section{Preliminaries}

Let $Y$ denote the observation process, which is the sum of signal and Gaussian noise, $Y=S+N$. Suppose that the process is defined on a bounded continuous-parameter domain $D$. The signal $S$ and the white noise $N$ are supposed to be independent processes (or operators), mapping the finitely additive probability space (or cylinder probability space) $\left(\mathcal{H}, C, \mu_{G}\right)$ into $(\mathcal{H}, C)$. Here, $\mathcal{H}=L_{2}^{n}(D)$, and $C$ the algebra of cylinder sets on $H . \mu_{G}$ is the finitely additive canonical Gaussian measure on $(H, C)$. The white noise process is equivalent to the identity operator, and is a weak random variable, which means that the measure induced by $N$ is again only finitely additive. The signal operator $S$ however is supposed to be a physical random variable which means that the measure $\mu_{S}$, induced by the signal can be extended to a countable additive measure over $(K, B)$ where $B$ is the Borel $\sigma$-algebra in $\mathcal{H}$. The observation process $\boldsymbol{Y}=\boldsymbol{S}+\boldsymbol{N}$ is again a weak random variable. For details on these definitions, see [7], [6].

Connected to a finitely additive measure $\mu$ in $(\mathcal{K}, C)$, one can define a covariance operator $R$. A measure can be extended to a countable additive measure on $(A, B)$ if and only if $R$ is nuclear. The covariance operator $R_{S}$ connected to the measure $\mu_{S}$ is in fact nuclear. $R_{S}$ is also said to be the covariance operator of the physical random variable $S$

A finitely additive measure $\mu_{1}$ is absolutely continuous with respect to another finitely additive measure $\mu_{2}$ if for any $\epsilon>0$, there exists a $\delta>0$ such that for any $C \in C, \mu_{2}(C)<\epsilon$ implies that $\mu_{1}(C)<\delta$. A finitely additive measure $\mu$ is absolutely continuous with respect to the canonical Gaussian measure $\mu_{G}$ if and only if the covariance operator of $\mu$ can be written as $I+R_{S}$ where $R_{S}$ is a Hilbert Schmidt operator. This implies that the measure induced by the observation process $Y, \mu_{Y}$ is absolutely continuous with respect to $\mu_{G}$ since it has covariance operator $I+R_{S}$ where $R_{S}$ is the covariance operator of $\mu_{S}$ which is nuclear and hence Hilbert Schmidt.

$$
\begin{aligned}
& 3 \text { The likelihood ratio for } \\
& \text { continuous-parameter processes }
\end{aligned}
$$

Let $Y$ be the observation process defined on a bounded continuous (multi-parameter) domain $D$, and $Y$ mapping into $H=L_{2}(D)$. For observations inducing a countable additive measure on the image space, the likelihood ratio can be defined as the Radon-Nikodym derivative of the measure induced by the observation process, $\mu_{Y}$, with respect to some reference measure. The Radon Nikodym derivative exists if the measure $\mu_{Y}$ is absolutely continuous with respect to this reference measure.

In finitely additive white noise theory, however, the observation process does not induce a countable additive measure on the image space, and therefore we need a modification of the definition of Radon-Nikodym derivative. Let $\mu_{G}$ be the canonical Gaussian (finitely additive!) measure on $(X, C)$, and $C$ denote the cylinder algebra in $X$. The likelihood ratio is defined to be the RadonNikodym derivative between the measures $\mu_{Y}$ and $\mu_{G}$, formally

$$
\mathcal{L}(Y)=\frac{\partial \mu_{Y}}{\partial \mu_{G}}(Y)
$$

More precisely, the Radon-Nikodym derivative between finitely additive measures can be defined a the $\mu_{G}$ physical random variable $F: \not \mathcal{R} \rightarrow R$ such that for each monotone increasing sequence of finite dimensional projections $\left\{P_{k}\right\}$, strongly converging to identity,

$$
\mu_{Y}(C)=\lim _{k \rightarrow \infty} \int_{C} F \circ P_{k}(f) d \mu_{G}(f),
$$

uniformly in $C \in C$. Although this property should hold for any sequence of finite dimensional projections, strongly converging to identity, one may prove that it is sufficient to find a $\mu_{G}$-physical random variable, and only one sequence of finite dimensional projections, such that the above equality holds true. This gives the opportunity to define a suitable sequence of projections which enables determination of $F$, and hence the likelihood ratio.

Therefore, let $\mu_{Y}$ be the measure induced by the observation process. Then $\mu_{Y}$ is a Gaussian finitely additive measure with covariance operator $I+R_{S}$, where $R_{S}$ is nuclear. Let $\left\{\phi_{k}\right\}$ be the complete set of orthonormal eigenfunctions of the operator $R_{S}$ in $*$ such that $R_{S} \phi_{k}=\lambda_{k} \phi_{k}$ and $\left[\phi_{k}, \phi_{j}\right]_{x}=\delta_{k, j}$. Note that such a set always exists, since $R_{S}$ is a self-adjoint Hilbert-Schmidt operator. Define the sequence of projections $\left\{P_{k}\right\}$, 
$P_{k}$ being the projection on the (finite- dimensional) space spanned by the first $k$ eigenfunctions $\phi_{1} \cdots \phi_{k}$ of $R_{S}$. Since $R_{S}$ is nuclear, the sequence of projections converges strongly to identity,

$$
\lim _{k \rightarrow \infty}\left|P_{k} f-f\right|_{k}=0
$$

for all $f \in X$.

Define the variables $Y_{k}=\left[\phi_{k}, Y\right]_{X}$ mapping into $(R, B)$. Then $Y_{k}$ are $\mu_{G}$-physical random variables and hence induce a countable additive Gaussian measure in $(R, B)$ with mean $E Y_{k}$ and covariance $1+\lambda_{k}$. Moreover, the variables $\left\{Y_{k}\right\}$ are mutually independent. Defined on $R$, the likelihood ratio of the measure induced by $Y_{k}$ is given by

$$
\begin{aligned}
\mathcal{L}_{k}(Y)= & \left(1+\lambda_{k}\right)^{-1 / 2} \\
& \exp \left(-\frac{\left(Y_{k}-E Y_{k}\right)^{2}}{2\left(1+\lambda_{k}\right)}\right) \exp \left(Y_{k}^{2} / 2\right) .
\end{aligned}
$$

Define $F_{k}(Y)=\prod_{1}^{k} \mathcal{L}_{j}(Y)$. Then $F_{k}(Y)$ is the Radon-Nikodym derivative between the measures $\mu_{Y}$ and $\mu_{G}$ restricted to the cilinder algebra $C_{P_{k}}$ induced by $\boldsymbol{P}_{k}$, which means that

$$
\mu_{Y \mid C_{\boldsymbol{P}_{k}}}(C)=\int_{C} F_{k}(f) d \mu_{G \mid \mathcal{C}_{\boldsymbol{P}_{k}}}
$$

uniformly in $C \in C_{P_{k}}$. Using the fact that $\left\{P_{k}\right\}$ is a sequence, strongly converging to identity, it follows that

$$
\mu_{Y}(C)=\lim _{k \rightarrow \infty} \int_{C} F_{k}(f) d \mu_{G}(f),
$$

uniformly in $C \in C$. According to the definition of the Radon-Nikodym derivative for finitely additive measures, $F$ defined by $F=\lim F_{k}$ is a $\mu_{G}$ physical random variable, representing the likelihood ratio for the observation process $Y$, since $F \circ P_{k}=F_{k}$. This results in

$$
\begin{aligned}
& \mathcal{L}(Y)=\prod_{1}^{\infty} \quad\left(1+\lambda_{k}\right)^{-1 / 2} \\
& \exp \left(-\frac{\left(Y_{k}-E Y_{k}\right)^{2}}{2\left(1+\lambda_{k}\right)}\right) \exp \left(Y_{k}^{2} / 2\right) .
\end{aligned}
$$

Using properties of infinite summation and multiplication, and the fact that elements in $\mathcal{K}$ have finite energy, this expression may be rewritten as

$$
\begin{aligned}
\mathcal{L}(Y)= & \operatorname{det}\left(I+R_{S}\right)^{-1 / 2} \\
& \exp \left(\left[R_{S}\left(I+R_{S}\right)^{-1} Y, Y\right]_{\mathcal{X}} / 2\right),
\end{aligned}
$$

where $E Y$ is supposed to be zero [10].

Application of the result above to practical situations will cause problems, since computation of determinant and inverse of the covariance operator is needed. In the situation where the parameter domain is one- dimensional, the expression for the likelihood ratio may be rewritten into a form where only the filter operator for the signal $S$ is needed. In the multi-dimensional situation, assuming for simplicity that $D$ is rectangular, we can use multi-directional Krein factorisation in order to rewrite the expression for the likelihood ratio. The likelihood ratio then may be expressed in one-directional filters for the signal $S$, which means filtering in one direction, and smoothing in all other directions [10]. This method has been succesfully applied to a generalization of Wong's two-parameter model [11].

Another situation where we may rewrite the likelihood ratio in a computable form is the case where we assume that the domain $D$ is cyclic. Then, using Fourier transforms, the eigenvalues of the covariance operator may be explicitely computed, and therefore the likelihood ratio.

\section{The likelihood ratio for discrete- parameter procesges}

For discrete-parameter processes, defined on a bounded domain, detection of the likelihood ratio is straightforward, in the sense that we only have a finite number of observation points. Let $Y$ be the observation process, defined on the bounded discrete-parameter domain $D, Y$ mapping into $l_{2}(D)$. Suppose that $Y$ is Gaussian with covariance operator $I+R_{S}$, where $R_{S}$ is the covariance operator for the signal $S$. Since $D$ is discrete and bounded, $l_{2}(D)$ is finite dimensional, and automatically, $I, R_{S}$, and $I+R_{S}$ are nuclear. This ensures countable additivity of the Gaussian measures defined on the Borel $\sigma$-algebra in this space. Moreover, $\left(l_{2}(D), B\right)$ is isomorf to $\left(R^{n}, B\right)$ for some $n$, denoting the number of elements in $D$. Transforming the observation process to $R^{n}$ (for instance by taking the vector of all observations in lexicographic ordering), $Y$ becomes a Gaussian random variable mapping into $R^{n}$, with $n \times n$-covariance matrix $\left(I+R_{S}\right)$. The likelihood ratio for the observation process then is given by

$$
\begin{aligned}
\mathcal{L}(Y)= & \operatorname{det}\left(I+R_{S}\right)^{-1 / 2} \\
& \exp \left(-Y^{T}\left(I+R_{S}\right)^{-1} Y / 2+Y^{T} Y / 2\right) .
\end{aligned}
$$

When the number of observation points is relatively large, computation of determinant and inverse of the covariance matrix may demand considerable computational efforts. In certain circumstances, the expression may be simplified. If we assume the parameter domain $D$ to be cyclic, Fourier transforms may again help to find the eigenvalues of the covariance matrix. Even more convenient is the situation where we may assume that the stochastic model is Markov (in multi-parameter sense). In that case, the model for the process 
may be written as a Nearest Neighbor model [1], [2], [9]. The structure of the $\mathrm{NNm}$ can be used for simple computation of the covariance matrix's determinant and inverse [12].

\section{Approximation of the likelihood ratio}

In section 3 we mentioned that computation of the likelihood ratio for continuous parameter processes may cause problems, since determinant and inverse of the infinite dimensional covariance operator $\left(I+R_{S}\right)$ is needed. In some situations the expression for the likelihood ratio may be rewritten in computable forms, but what can be done in the general situation? The usual way is to try to find an approximation, and so we do. In this section we show how the finitely additive white noiuse setup gives a straightforward method for approximation of the likelihood ratio, using finite dimensional projections. In section 3 it was pointed out that the likelihood ratio for a process $Y$ defined on $\mathcal{X}$ is given by the unique $\mu_{G}$-physical random variable $F: \mathcal{H} \rightarrow R$ such that for each monotone increasing sequence of finite dimensional projections $\left\{P_{k}\right\}$, strongly converging to identity,

$$
\mu_{Y}(C)=\lim _{k \rightarrow \infty} \int_{C} F \circ P_{k}(f) d \mu_{G}(f)
$$

uniformly in $C \in C$.

The likelihood ratio hence is defined via finitedimensional projections, and this opens the way to approximate the likelihood ratio $\mathcal{L}$ by its finitedimensional counterpart $\mathcal{L} \circ P_{k}$.

Let $\left\{P_{k}\right\}$ be an arbitrary sequence of finitedimensional projections, monotonically increasing, and strongly converging to identity. Let $\tilde{Y}^{k}$ be the projection of $Y$ on $\mathcal{H}_{P_{k}}$, the image space of the projection $P_{k}$. Let $\tilde{Y}^{k}=P_{k} \circ Y$, and $\mathcal{L}^{k}\left(\tilde{Y}^{k}\right)$ the likelihood ratio for the model $\tilde{Y}^{k}=\tilde{X}^{k}+\tilde{N}^{k}$. Theorem 5.1 The likelihood ratio for the projected model converges to the likelihood ratio for the original continuous parameter model if $k$ tends to infinity in the sense that for and $\epsilon>0$,

$$
\lim _{k \rightarrow \infty} \mu_{G}\left(\mid \mathcal{L}(Y)-\mathcal{L}^{k}\left(\tilde{Y}^{k}\right)\right.
$$

\section{Proof}

The Radon-Nikodym derivative $F$ between $\mu_{Y}$ and $\mu_{G}$ is a $\mu_{G}$ physical random variable. This implies that $F$ satisfies the property that for all $\epsilon>0, \lim _{k \rightarrow \infty} \mu_{G}\left(\left|F \circ P_{k}-F\right|>\epsilon\right)=0$. Since $\mu_{Y}$ is absolutely continuous with respect to $\mu_{G}$, this also implies that $\lim _{k \rightarrow \infty} \mu_{Y}\left(\left|F \circ P_{k} F\right|>\epsilon\right)=0$, and $\lim _{k \rightarrow \infty} \mu_{G}\left(\left|F \circ P_{k}(Y) F(Y)\right|>\epsilon\right)=0$ (note that $Y$ maps $\left(K, C, \mu_{G}\right)$ into $\left.\left(X, C, \mu_{Y}\right)\right)$. Now, by definition, $\mathcal{L}(Y)=F(Y)$ which proves the theorem.
The theorem proves, that any sequence of finitedimensional projections, strongly - converging to identity, gives a sequence of likelihood ratios, converging to the likelihood ratio for the continuousparamter stochastic process. This implies that approximation for instance holds for Fourier sequences, wavelets, etcetera. In next section we show how discretisation also fits in this setup.

\section{Discretization}

Discretisation of the stochastic process is one method to approximate the likelihood ratio for the original continuoue-parameter stochastic process. The feature of this method is that many results are known for determination of the likelihood ratio for discretized models.

In this section we will restrict to the case where the parameter domain $D$ is two-dimensional and a rectangle, $D=\left[0, T_{1}\right] \times\left[0, T_{2}\right]$. Extensions to non-rectangular and general multi-parameter processes can be easily made. Very nice theoretical results are obtained for Nearest Neighbor models, see for instance [12] and [9]. Discretization of a stochastic process governed by an elliptic partial differential equation leads to a NNm. Since it turns out that discretization leads to an unbiased approximation for the likelihood ratio, it may well be used for estimation of unknown (distributed) parameters in the model for the process.

In order to derive that discretization is equivalent to finite-dimensional projection, we introduce a partition of $D$ in parts

$$
D_{\underline{j}}^{k}=\left[\frac{j_{1} T_{1}}{2^{k}}, \frac{\left(j_{1}+1\right) T_{1}}{2^{k}}\left[\times\left[\frac{j_{2} T_{2}}{2^{k}}, \frac{\left(j_{2}+1\right) T_{2}}{2^{k}}[\right.\right.\right.
$$

for $\left(j_{1}, j_{2}\right) \in\left\{0, \ldots, 2^{k}-1\right\}^{2}$. Let $h_{1}^{k}=\frac{T_{1}}{2^{k}}$ and $h_{2}^{k}=\frac{T_{2}}{2^{k}}$ be the horizontal and vertical stepsizes. Now define the set of orthonormal functions $g_{\underline{j}}^{k} \in$ $L_{2}^{n}(D)$ as

$$
g_{\underline{j}}^{k}(\underline{t})=\frac{1}{\sqrt{h_{1}^{k} h_{2}^{k}}} 1_{D_{i}^{k}}(\underline{t})
$$

where $1_{D_{i}^{k}}$ is the indicator function. Let $P_{k}$ be the projection operator on the finite-dimensional space $\varkappa_{P_{k}}$ spanned by the functions $g_{j}^{k}$. Then the space $\mathcal{H}_{P_{k}}$ denotes the set of square integrable step functions with horizontal step size $h_{1}^{k}$ and vertical step size $h_{2}^{k}$. Further, the sequence of projections $\left\{P_{k}, k=1, \ldots\right\}$ is monotonically increasing and strongly converging to identity, which implies that this sequence may be used for approximation of the likelihood ratio. Since the functions $g_{i}^{k}$ span $\mathcal{K}_{P_{k}}$ and are orthonormal in $L_{2}(D)$, the projection $P_{k}$ is defined by

$$
P_{k}(f)=\sum_{(i, j) \in\left\{0, \ldots, 2^{k}-1\right\}^{2}}\left[g_{\underline{j}}^{k}, f\right] g_{\underline{j}}^{k}
$$


Note that this projection is equivalent to the discretization $G^{k}: L_{2}^{n}(D) \rightarrow l_{2}^{n}\left(\left\{0, \ldots, 2^{k}-1\right\}^{2}\right)$,

$$
\begin{aligned}
\tilde{f}^{k}(\underline{j}) & =G^{k}(f)(j) \\
& =\left[g_{\underline{j}}^{k}, f\right] \\
& =\frac{1}{\sqrt{h_{1}^{k} h_{2}^{k}}} \int_{D_{i}^{k}} f(t) d \underline{.}
\end{aligned}
$$

One may easily verify that $G^{k}$ indeed maps into the space of square summable functions.

In the case that we restrict ourselves to the continuous functions, $C^{n}(D) \subset L_{2}^{n}(D)$, unbiasedness of the approximation also holds for the situation where we obtain discretiration via sampling.

\section{Concluding Remarks}

In this paper we derive approximations for the likelihood ratio for continuous-parameter stochastic processes. We may conclude that any sequence of strocgly converging finite-dimensional projections provides an unbiased approximation. Further research has to be done on the problem which kind of projections are most suitable for particular problems, in the sense that the approximation is efficient. Another question is how things work out for some particular problems, like the Poisson equation, etc.

\section{References}

[1] M.B. Adams, A.S. Willsky and B.C. Levy (1984). Linear Eatimation of Boundary Value Stochastic Processes - Part 1: The Role and Construction of Complementary Models. IEEE Transactions on Automatic Control 29, pp. 803-811.

[2] M.B. Adams, A.S. Willsky and B.C. Levy (1984). Linear Estimation of Boundary Value Stochastic Processes - Part 2: I-D Smoothing Problems. IEEE Transactions on Automatic Control 89, pp. 811-822.

[3] A. Bagchi (1985). Cylindrical Measures in Hilbert Space and Likelihood Ratio for TwoParameter Signals in Additive White Noise. Control Theory and Advanced Technology 1, pp. 139-153.

[4] A. Bagchi and R. Masumdar (1990). Direct Modeling of White Noise in Stochastic Systems. In Topics in Stochastic Systems: Modelling, Estimation and Adaptive Control ( $L$. Gerenscser, P.E. Caines, Ede.) Lecture Notes in Control and Information Sciences 161, Springer Verlag, New York, pp. 1-29.
[5] A.V. Balakrishnan (1980). Nonlinear White Noise Theory. In Multivariate Analysis-V (P.R. Krishnaiah, Ed.), North Holland Publishing Company, Amsterdam.

[6] A.V. Balakrishnan (1981). Applied Functional Analysis, second edition, Springer Verlag, New York.

[7] G. Kallianpur and R.L. Karandikar (1985). A White Noise Calculus and Nonlinear Filtering Theory. Special invited paper in Annals of Probability Theory 19, pp. 1033-1107.

[8] G. Kallianpur and R.L. Karandikar (1988). White Noise Theory of Filtering, Prediction and Smoothing. Gordon and Breach Publishers, London.

[9] B.C. Levy (1990). Noncausal Estimation for Discrete Gauss Markov Random Fields. In Realization and Modelling in System Theory, Proceedings of the International Symposium MTNS-89, Amsterdam, Volume 1 (M.A. Kaashoek, J.H. van Schuppen and A.C.M. Ran, Eds.), Birkhauser, Boston, pp. 13-21.

[10] R. Luesink and A. Bagchi (1991). Krein Factorisation of Covariance Operators of TwoParameter Random Fields and Application to the Likelihood Ratio. IEEE Transactions on Information Theory S7, pp. 53-59.

[11] R. Luesink and A. Bagchi (1991). The Likelihood Ratio for Two Dimensional Stochastic Processes. In Proceedings 1st European Control Conference ,Grenoble, France, pp. 608612.

[12] R. Luesink (1992). On the Likelihood Ratio for Two-Parameter Discrete Space Stochastic Processes. Reports in Operations Research and System Theory, M92-05, University of Limburg. Submitted for publication. 